\title{
Ion Trajectories in an Electrostatic Ion Guide for External Ion Source Fourier Transform Ion Cyclotron Resonance Mass Spectrometry
}

\author{
Jarrod A. Marto* and Alan G. Marshall \\ Department of Chemistry. The ()ho Stote Lniversity, Columbus, (ohiu, Usi \\ Michael A. May \\ Department of Chemistre, Central State Lniversits, Wilberforec, (Bhio, LSA
}

Patrick A. Limbach

Department of Chemistry, I ouisiance State L niversity, Baton Rouge, I oulisiand, LSA

\begin{abstract}
An electrostatic ion guide (EIG) that consists of concentric cylinder and central wire electrodes can transport ions efficiently from an external ion source to an ion cyclotron resonance (ICR) ion trap for mass analysis, with several advantages over current injection methods. Because the electrostatic force of the EIG captures ions in a stable orbit about the wire electrode, ions with initially divergent trajectories may be redirected toward the ICR ion trap for improved ion transmission efficiency. SIMION trajectory calculations (ion kinetic energy, $1-200 \mathrm{eV}$; elevation angle, 0 30; azimuthal angle, $0-360^{\circ}$ ) predict that ions of $\mathrm{m} / \mathrm{z}$ 1000 may be transmitted through a strong $(0.01 \rightarrow 3.0$-T) magnetic field gradient. Judicious choice of ion source position and EIC potential minimizes the spread in ion axial kinetic energy at the ICR ion trap. Advantages of the EIC include large acceptance angle, even for ions that have large initial kinetic energy and large radial displacement with respect to the central z-axis, low ion extraction voltage $(5-20 \mathrm{~V})$, and efficient trapping because ions need not be accelerated to high velocity to pass through the magnetic field gradient. (f Am Soc Mass Spectrom 1995, 0, 936-940)
\end{abstract}

$\mathrm{T}$ he performance adiantages of Fourier transform ion cyclotron resonance mass spectrometry (FT$\mathrm{ICR} / \mathrm{MS})$, which include high mass resolution, high mass accuracy, and long ion storage period [1-13], are optimized by high magnetic field strength $(>3 \mathrm{~T})$ and low pressure $\left(<10^{\prime}\right.$ torr) in the ion cyclotron resonance (ICR) ion trap. Because' some useful ionization sources (e.g., fast-atom bombardment, glow discharge) do not operate conveniently under such conditions, optimal Fourier transform ion cyclotron resonance (FT-ICR) performance mav require "external" ion generation with subsequent ion injection into the ICR ion trap. The problems associated with external ion injection and the gencral approaches currently used have been discussed previously in some detail [1, 14-18]. We previously proposed and demonstrated experimentally the use of an electrostatic ion guide

Address reprint requesto to l'rofensor Man (i. Marshall. Center for Interdiceiplinary Magnetic Resennance, Votional I ligh Magnetio [ieded Laboratory, Florida State Universits, Isole fast Pab] Dirac Drive Tallahasises, I'L 32310 .

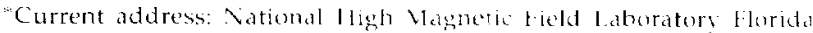
State University, 1800 F. Paul Dirac Drive, Tallahassee, IL 32310.

Member of the Department at Chemistru, Florida State Linibersits.
(EIC), which consists of a cylinder and central wire held at different d.c. potential, to generate an electrostatic radially inward-directed force sufficiently strong to guide ions through a magnetic field gradient and into an ICR ion trap [14].

We first demonstrated the EIG operated with a thermionic emitter source external to a solenoidal 3-T magnet. Subsequently, we have coupled an external matrix-assisted laser desorption/ionization (MALDI) and $\mathrm{Cs}^{+}$secondary ion mass spectrometry (SIMS) source [19] and an rf glow discharge source [20] to an FT-ICR mass spectrometer by use of an intervening FIG. Finally, we demonstrated that broadband axialization (produced by azimuthal quadrupolar storedwaveform inverse Fourier transform (SWIFT) excitation in the presence of pulsed collision gas] greatly enhances the trapping efficiency, signal-to-noise ratio, and mass resolving power for ions injected from both external SIMS and MALDI sources through an EIG to an ICR Penning trap [17].

The principles of operation of an EIG in the absence of a magnetic field have been described previously [21-30]. For ion injection in FT-ICR/MS, the EIG equations of motion are further complicated by the pres- 
ence of a magnetic field gradient. Here, we first discuss the equations of motion for a infinitely exterkled EIG in the absence of a magnetic field. We then employ numerical trajectory calculations to characterize transport by an electrostatic ion guide of ions (1-200-e V initial kinetic energy) axially through the strong magnetic field gradient $(0-3 \mathrm{~T})$ of an FT-ICR mass spectrometer. Specifically, we consider the effects of ion kinetic energy, EIG voltages (absolute and difference), ion source placement, and initial ion angular distribution on both ion transport efficiency and ion axial kinetic energy.

\section{Theory: Analytical Methods}

We begin by presenting analytical expressions relevant to the EIG, for an ion point source or circular disk source located in the entrance plane of the guide. We then offer numerical simulations that correspond to typical experimental conditions, for an ion point source at various locations in (or displaced axjally from) the EIG entrance plane. The intended function of the EIC; is to transport ions from an externally located ion source into an ICR ion trap without any additional acceleration, focusing, and/or deceleration lenses. Wo assume that an ion source can generate a large flux of ions at the EIG entrance plane and that the ions have? kinetic energies between 1 and $200 \mathrm{eV}$ (see Figure 1 for coordinate system and ion guide confjururation and dimensions).

Electrostatic Ion Guide in the Alsence of a Magnetic Ficld

The electromagnetic force that acts on an ion of mass $m$ and charge $q$ is given by the lorent 2 force equation:

$$
\left.\left.\mathbf{F}=m \mathbf{a}={ }_{17} \mathbf{E}+(1\} \mathbf{v} \times \mathbf{B}\right) \text { (S.). units }\right)
$$

in which $\mathbf{a}$ is ion acceleration, $\mathbf{v}$ is ion velocity, $E$ is electric field, and $\mathbf{B}$ is magnetic induction. The axial and radial electric field magnitudes $E$ and $E$, between two infinitely extended concentric cylinders (i.e., an idealized electrostatic ion guide) may be expressed (in cylindrical coordinates) as

$$
\begin{aligned}
& E_{z}=0 \\
& E_{r}=-\frac{\Delta V}{r \ln \left(r_{\text {(s) }} / r_{\text {(nlinder }}\right)}
\end{aligned}
$$$$
(2,3)
$$

in which $E$, is the axial electric field, $r$, is the radial electric field, $r$ is the radial displacement of the ion from the central axis, $J V=V_{\text {...lind.r }}-V_{\text {wire }}$ is the portential difference between the outer and central cylinders, $r$ culinder is the radius of the outer cylinder, and $r_{\text {wire }}$ is the radius of the central wire.

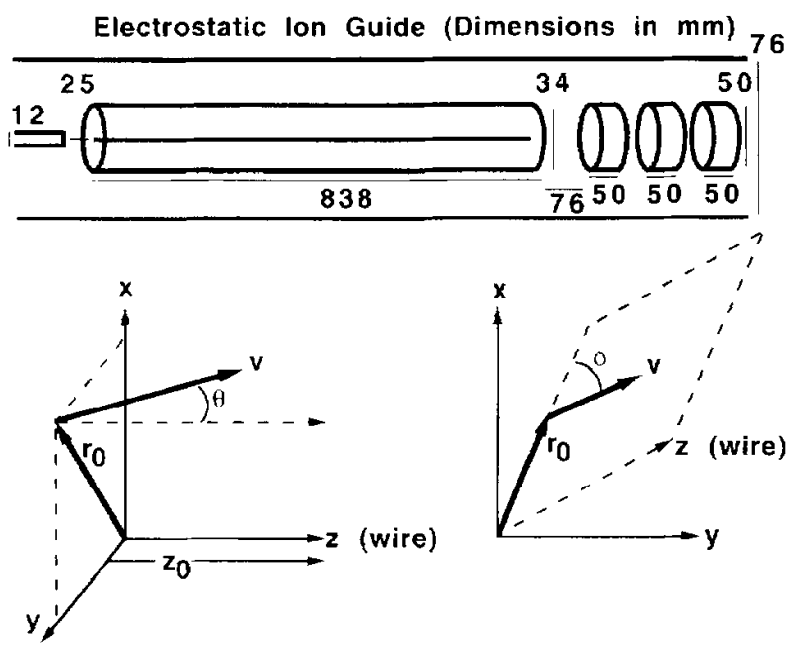

Figure 1. Schernatic diagram of the electrostatic ion guide (EIG) configuration, with dimensions shown in millimeters. lop: The thre' maior electrode sets, which include the source, the EIC wire and rylinder, and the ICR Penning trap. Bottom: Coordinate sustem on which eqs $7-12$ are based. The ion source is located at $i_{1}, z_{11}$ and the ion initial velocity defines an "elevation" angle $\theta$ and an "aximuthal" angle" do as shown (see text).

\section{Magnetic Mirror Effect in the Absence} of an Electric Field

The magnitude of the axial magnetic field $B$ : along the central axis of a solenoidal electromagnet may be approximated as

$$
B=\frac{\mu_{i} N J r_{\text {(ithendid }}^{2}}{2} \int_{-12\left(r_{\text {silendid }}^{2}+z^{2}\right)^{3 / 2}}^{1}
$$

in which $B$ is the magnetic field near the $z$ axis, $\mu_{0}$ is the magnetic permittivity of a vacuum, $N$ is the number of lurns of the solenoidal conductor per unit length, $I$ is the current in a single loop of the solenoid, $L$ is the solenoid length, $=$ is the axial displacement of the ion with respect to the center of the solenoid, and $r_{\text {solemoid }}$ is the solenoid radius. Because the radial and axial magnetic ficld components $B(r)$ and $B(z)$ are related to each other by Maxwell's equation $\nabla \cdot \mathbf{B}=0$, the magnitude of the radial magnetic field $B_{r}$ also may be estimated near the solenoid central axis [31]:

$$
\begin{aligned}
& \frac{1}{r} \frac{\partial}{d r}\left[r B_{r}\right]+\frac{\partial B_{z}}{d z}=0 \\
& B_{r} \approx-\frac{r}{2}\left[\frac{d B}{d z}\right]_{r-1}
\end{aligned}
$$

(Fquation 5 is approximately valid in the limit that $\mathrm{H} B . / \mathrm{d}$ does not vary strongly with $r$.) Thus, the radial magnetic field magnitude is strongest when the axial magnetic field gradient is strongest - for our 3-T magnet, at $=25 \mathrm{~cm}$ from the center of the solenoid. The radial magnetic field $B$ of eq 5 gives rise to the well known "magnetic mirror" effect. An ion that has a velocity component ' ' perpendicular to the $z$ axis 
(and thus perpendicular to $B_{r}$; see succeeding text) will experience a force whose axial magnitude $F_{z}$ is

$$
F_{z}=q 7 \cdot B
$$

Because $B_{r}$ reaches a maximum when $B_{z}$ is about half of its maximum value, $\bar{c}$, at that $z$ position is essentially perpendicular to $B_{r}$, because $v$ is dominated by ion cyclotron motion about $B_{-}$, and the ( $q v^{\prime} B_{1}$ ) force is therefore always in the negative $z$ direction (i.e., a retarding force that reduces the ion $z$ velocity). (Note that this argument applies whether $B$ = points along the positive or negative $z$ direction, because the sign of $\left[d B_{z} / d z\right]_{r}, n$ changes if the direction of $B$. changes.) Thus, the "magnetic mirror" slows (off-axis) ions as they approach a region of strong magnetic field.

\section{Electrostatic Ion Guide in a Magnetic Field}

Substitution of eqs 2, 3, and 5 into eq 1 yields the equations of motion for an ion inside an infinitely extended electrostatic ion guide and near the axis of a solenoidal magnet. Lnfortunately, an analytical solution to eq 1 is not possible because there is no analytical expression for the electric or magnetic ficld over the course of ion travel from the ion source to the FT-ICR ion trap (see Figure 1). Therefore, we resort to numerical calculations (see Results and Discussion) to establish the approximate initial conditions that favor ion capture and transport through the magnetic field gradient and into an ICR ion trap.

It is interesting to note that a central-wire ion guide to which electrically charged "end caps" are added is known as a "Kingdon" ion trap, which originally was described in 1923 [32]. Johnson has discussed the operation of a Kingdon trap in a static magnetic ficld [33].

\section{Analytical Expressions: Electrostatic Ion Guide in the Absence of a Magnetic Ficld}

Typically, an external ion source is located far $(>1 \mathrm{~m})$ from the center of the solenoid of an FT-ICR mass spectrometer. The magnetic field at the source is thus negligible and ion behavior in this region (e.g., at the entrance of the EIG) may be estimated from analytical expressions (see eqs $7-12$ ). Let the EIG axis be collinear with the solenoidal magnetic field symmetry $=$ axis, and let the ion (point) source be displaced radially (by $r_{0}$ ) and axially (by $z_{0}$ ) from the center of the EIG entrance plane (see Figure 1). Let the initial ion velocity $\mathbf{v}$ define an "elevation" angle $\theta$ relative to a line that contains the initial position coordinates of the ion and is parallel to the $=$ axis. In addition, there is an "azimuthal" angle $\phi$ between the projection of $\mathbf{v}$ onto the $x y$ plane and a plane that contains both the ion initial position and the $z$ axis. Based on the preceding coordinate system, with $z_{01}=0$ ite.e, ion source in the entrance plane of the EIG), Oakey and Macfarlane [23] derived the following expression for the ion maximum "acceptance cone angle" for ions that enter an infinitely extended EIG in the absence of a magnetic ficld:

$$
\theta_{\text {max }}^{2}=\frac{q|\Delta V|}{E_{\mathrm{ion}}} \frac{\left[\ln \left(r_{0} / r_{\text {colinder }}\right) / \ln \left(r_{\text {wire }} / r_{\text {cylinder }}\right)\right]}{\left[1-\left(r_{0}^{2} / r_{\text {cylinder }}^{2}\right) \sin ^{2} \phi\right]}
$$

in which $\theta_{\max }$ is the maximum "elevation" angle at which ions may still enter and successfully traverse the EIG and $E_{\mathrm{ion}}$ is the ion kinetic energy [23]. Oakey and Macfarlane [23] have derived the equations for the "collection efficiency" of either a point source or a circular disk source, both located at the entrance plane of the EIG. The collection efficiency $\operatorname{Eff}_{\text {point }}\left(r_{0}\right)$ for a point source at $r_{0}$ in the EIG entrance plane is defined [23] as the solid angle of the acceptance cone divided by $4 \pi$ :

$$
\begin{aligned}
\operatorname{Eff}_{\text {puint }}\left(r_{0}\right)= & \frac{0.25 q|\Delta V|}{E_{\mathrm{i}, \mathrm{n}}} \\
& \times \frac{\ln \left(r_{0} / r_{c y \text { linder }}\right) / \ln \left(r_{\text {wire }} / r_{\text {cylinder }}\right)}{\left[1-\left(r_{0}^{2} / r_{\text {cylinder }}^{2}\right)\right]^{1 / 2}}
\end{aligned}
$$

Similarly, the collection efficiency from a circular diskshaped source of radius $r_{\text {disk }}$ (for $r_{\text {disk }} \gg r_{\text {wire }}$ ) is

$$
\begin{aligned}
& \operatorname{Eff}_{\text {disik }}\left(r_{\text {disk }}\right) \\
& =\left(\frac{0.5 q|\Delta V|}{E_{\mathrm{i} e n} \ln \left(r_{\text {wire }} / r_{\text {ollinder }}\right)} \frac{r^{2}}{r_{\mathrm{di} \text { ilinder }}^{2}}\right) \\
& \times\left[\left[1-\ln \left(r_{\text {disk }} / r_{\text {cylinder }}\right)\right]\left(1-\frac{r_{\text {disk }}^{2}}{r_{\text {cylinder }}^{2}}\right)^{1 / 2}\right. \\
& \left.-\ln \left(1+\left(1-\frac{r_{\text {disk }}^{2}}{r_{\text {cylinder }}^{2}}\right)^{1 / 2}\right) \frac{r_{\text {cylinder }}}{r_{\text {disk }}}\right) \\
& -0.3069]
\end{aligned}
$$

As seen in eq 7 , the potential difference $\Delta V$ between the wire and cylinder and initial kinetic energy $E_{\mathrm{i} \text { m }}$ determine whether an ion attains a stable orbit about the central wire. Thus, for a fixed EIG geometry, ions of widely varying energy may be transported successfully, simply by adjusting the potential difference between the wire and cylinder electrodes. Conversely, monoenergetic ions of different mass should be readily captured by the EIG at a given voltage. Furthermore, lower energy ions have a larger acceptance cone, which makes the EIG particularly well suited for typical ion sources used in FT-ICR mass spectrometry. Also, the direct dependence of maximum acceptance cone angle and collection efficiency 
on ion charge means that multiply charged ions (as from an electrospray ion source) should be captured more readily by the EIG for transport into the ICR trap.

\section{Theory: Computational Methods}

\section{SIMION Numerical Simulations: Electrostatic Ion Guide in a Magnetic Field Gradient}

We now proceed to examine EIG performance systematically as a function of $J V, E_{i, n}, r_{0}, H, \phi$, and $=$ displacement of the ion (point) source away from the entrance plane of the EIG, for a particular ion mass-tocharge ratio $m / z$. Figure 1 shows the geometry and nominal dimensions of an EIG, previously demonstrated in our lab [17], on which all numerical simulations were based. The guide cylinder is $838 \mathrm{~mm}$ long, with $34-\mathrm{mm}$ inside diameter. The central wire is 838 mm long and $0.28 \mathrm{~mm}$ in tiameter, and is aligned coincident with the central symmetry $(z)$ axis of the solenoidal magnet. A cylindrical ion source electrode biased at $+5-\mathrm{V}$ potential was positioned at various points in or displaced axially from the ion guide entrance plane. A three-section open-ended cylindrical ICR ion trap was centered in the solenoidal magnet, 70 $\mathrm{mm}$ axially displaced from the ion guide exit. Each ICR trap section was $50 \mathrm{~mm}$ long and $50 \mathrm{~mm}$ in diameter, and each was centered on the $z$ axis. The vacuum chamber that surrounds the ion source, ion guide, and ICR ion trap was set to ground potential for all calculations. In all numerical simulations (e.g., Figures 4 9), ion initial conditions are designated with respect to a point source electrode, where $r_{11}$ still denotes the radial displacement of the source probe. (Note that eqs 7-12, Tables 1 and 2, and Figure 3 refer to an ion source in the entrance plane of the EIG.)

Ion trajectory simulations were performed with SIMION PC/PS2 (Version 5.0, Idaho National Engineering Laboratory, Idaho Falls, ID). All calculations were based on a two-dimensional electric potential array of $600 \times 40$ points; a three-dimensional array was then generated by assuming cylindrical symme try. Magnetic field plots for s 3- $T$ superconducting magnet were provided by Oxford Instruments Inc. (Scientific Research Division, Concord, MA). Discrete values for both $B$ and $B$, were supplied at $5-\mathrm{cm}$ axial increments $(0-110-\mathrm{cm}$ axial displacement from the solenoid center) and $0.5-\mathrm{cm}$ radial increments $(0-3-\mathrm{cm}$ radial displacement from the solenoid central axis). Best-fit lines for $B=(r=0 \mathrm{~cm}), B,(r=1 \mathrm{~cm}), B,(r=2$ $\mathrm{cm})$, and $B_{r}(r=3 \mathrm{~cm})$ are plotted versus axial displacement $(z)$ from the solenoid center in Figure 2.

Discrete values of $B:(r=0 \mathrm{~cm})$ and $B,(r=3 \mathrm{~cm})$ were entered into a magnetic potential array in SIMION at $5-\mathrm{cm}$ axial increments, beginning at the ion trap center and extending to the ion source probe tip $(0 \leq=$ $\leq 110 \mathrm{~cm}$ ). During trajectory calculations SIMION used linear interpolation to obtain $B$, and $B$, at ion positions other than those that correspond to the discrete

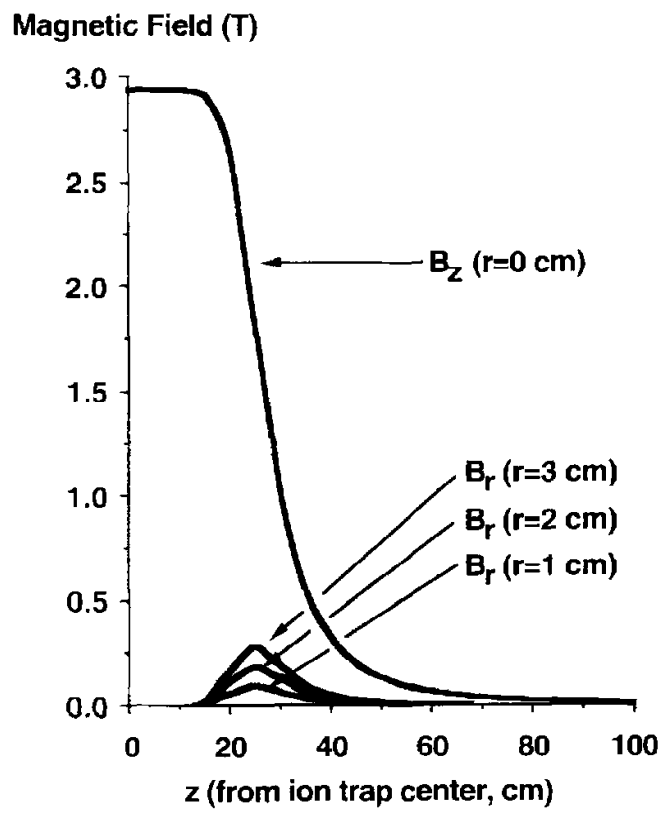

Figure 2. Axial ( $z$ component) and radial ( $r$ component) magnetic field magnitudes $B_{z}$ and $B_{r}$ as input parameters to SIMION for our 3-T solenoid magnet. The magnetic field value between any two $B$ and $B$, points (entered at $5-\mathrm{cm}$ increments) is obtained by linedar interpolation.

magnetic field entries. Note that values for $B_{z}(r=1-3$ $\mathrm{cm})$ agree very well with those for $B=(r=0 \mathrm{~cm})$, which indicates that, in fact, $d B_{z} / d z$ does not vary strongly with radius (up to $3 \mathrm{~cm}$ ). Thus eq 5 holds and our use of linear interpolation to estimate $B_{r}$ at radii between 0 and $3 \mathrm{~cm}$ introduces only minimal error. Likewise, linear interpolation will slightly overestimate $B$, at large axial displacements ( $\geq 40 \mathrm{~cm}$; see Figure 2) while slightly underestimating $B$ z at axial displacements of $-10-22 \mathrm{~cm}$ (e.g., between the regions of maximum axial and radial magnetic fields; see Figure 2). More important, in the region of maximum

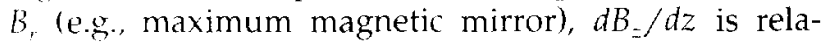
tively constant, so that linear interpolation provides accurate estimates of $B$. .

Because ionization sources produce ions with both low (e.g., glow discharge, electrospray) and high (e.g., MALDI) kinetic energies, we computed trajectories for ions that have initial kinetic energies in the (broad) range $1-200 \mathrm{eV}$. The partitioning of that ion energy in different directions is then fixed by specification of the initial angular divergence in our ion trajectory simulations.

\section{Results and Discussion}

\section{Ion Acceptance into the Electrostatic Ion Guide}

If an ion is to pass through the EIG, it is obvious that it cannot collide with either of the two electrodes. An ion that enters the EIG at greater than a maximum allowable elevation angle $\theta_{\text {max }}$ will collide with the outer 
electrode. Moreover, an ion that enters the guide must have a minimum angular momentum to avoid collision with the central wire.

Figure 3 is a plot of the collection efficiency (computed from eq 8) as a function of ion initial off-axis displacement $r_{0}$ for four combinations of ion initial kinetic energy and EIG potential difference $\Delta V$ for a point ion source in the entrance plane of the EIG. As expected, the collection efficiency for ions to be captured in a stable orbit in the EIG for transport to the ICR ion trap increases with increasing ratio of potential difference to ion initial kinetic energy $\Delta V / E_{\text {ion }}$. It is encouraging to find that a significant fraction of ions that enter the EIG significantly off-axis (large $r_{0}$ ) is captured by the guide (recall that eq 8 applies to a point source that emits ions over $4 \pi$ geometry). Table 1 shows that expanding the ion source from a point to an on-axis circular disk of $6-\mathrm{mm}$ radius increases the absolute collection efficiency still further. However, the ratio $\Delta \mathrm{Eff}\left(r_{\text {disk }}\right) / \Delta r_{\text {disk }}$ decreases with increasing disk radius. In other words, for a circular disk ion source, each incremental increase in ion source surface area results in a smaller incremental increase in collection efficiency. Our simulations indicate that the energy dependence (eq 7) for successful ion capture also holds for ions formed outside the EIG; for example, when the source probe is displaced by $6 \mathrm{~mm}$ with respect to the EIG entrance plane, monoenergetic ions over the range $50 \leq m / z \leq 100,000\left(r_{0}=2 \mathrm{~mm}, \theta=15^{\circ}, \phi=45^{\circ}, \Delta V\right.$ $=10 \mathrm{~V}, E_{\mathrm{ion}}=20 \mathrm{eV}$ ) all had maximum Lissajous orbital radii (e.g., ion apogee radius inside the EIG) of $\sim 4.4 \mathrm{~mm}$ and were transported to the ICR ion trap.

As the axial displacement of a point ion source from the EIG increases, the maximum allowed ion kinetic energy decreases. Figure 4 shows that an ion that begins axially near the EIG with an elevation angle $\theta=15^{\circ}$ has an increased probability of attaining a stable trajectory inside the EIG, mainly because a

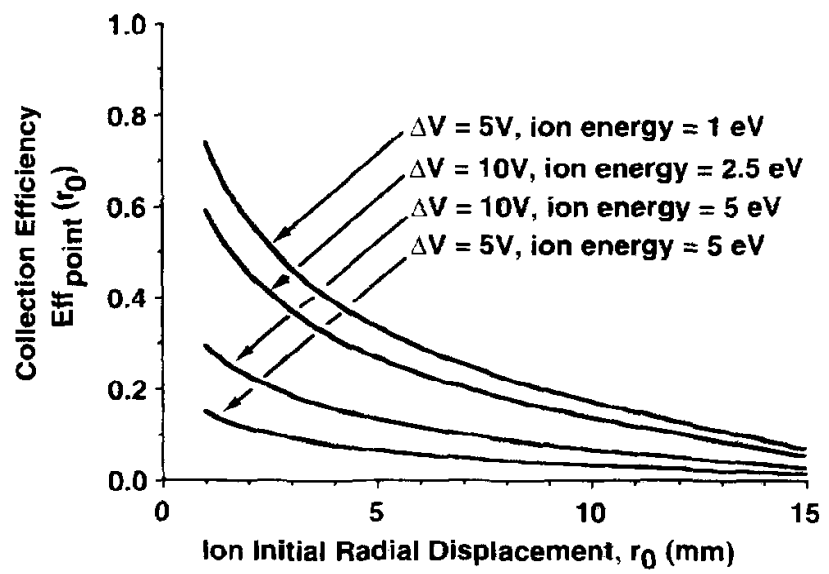

Figure 3. Collection efficiency Eff point $\left(r_{0}\right)$ of a point ion source located in the entrance plane of the EIG as a function of the ion source radial displacement $\gamma_{0}$. Note that even ions initially far off-axis are successfully captured and transported by the EIG to the ICR Penning trap.

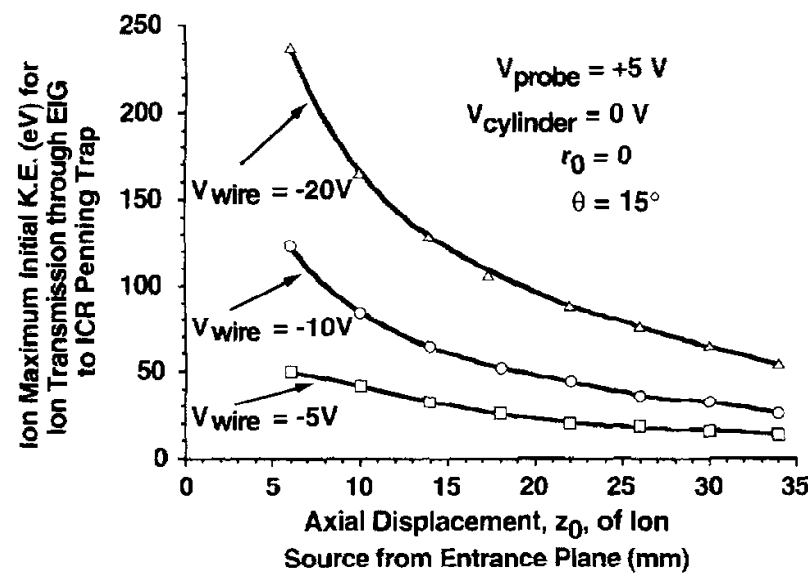

Figure 4. Maximum ion kinetic energy for EIG capture and ion transfer to an ICR Penning trap versus initial $z$ displaccment $\left(z_{0}\right)$ of the on-axis $\left(r_{0}=0\right)$ ion source from the EIG entrance plane, for an ion of $m / z 1000$, at EIG potential differences $\Delta V=5,10$, or $20 \mathrm{~V}$. The ion initial velocity elevation angle $\theta$ is fixed at $15^{\circ}$. Note that an ion that starts out closer to the EIG entrance may be accepted at a higher kinetic energy, for increased coliection efficiency.

smaller initial axial displacement results in a smaller initial radial displacement $r_{0}$ at the entrance plane: in eq $8, \ln \left(r_{0} / r_{\text {cylinder }}\right) / \ln \left(r_{\text {wire }} / r_{\text {cylinder }}\right) \rightarrow 1$ as $r_{0} \rightarrow r_{\text {wire }}$ (i.e., as the ion initial radial displacement approaches the central wire). Stated another way, if the ion source is moved closer to the EIG, then the same number of ions can be collected by an EIG of smaller outer cylinder radius $r_{\text {cylinder }}$. Figure 4 also confirms that a higher cylinder-wire potential difference increases the probability of a stable orbit inside the guide.

Figure 5 shows how the maximum initial kinetic energy for which ions of $\mathrm{m} / \mathrm{z} 1000$ still may be captured by the EIG depends on initial polar ("elevation") entry angle $\theta$ for fixed initial axial and radial displacements, again for each of three values of EIG potential difference. For example, for an EIG potential difference of $10 \mathrm{~V}$, an ion with an initial elevation angle of $20^{\circ}$ and up to $\sim 43-\mathrm{eV}$ initial kinetic energy still will be captured by the guide and transported to the ICR Penning trap. The maximum kinetic energy decreases to $\sim 18 \mathrm{eV}$ at $30^{\circ}$ and $\sim 8 \mathrm{eV}$ at $40^{\circ}$, in qualitative agreement with eq 7 , which predicts that $E_{\text {ion }}$ should vary as $\left(\theta_{\max }\right)^{-2}$.

Equation 7 also predicts how ion capture depends on the azimuthal entry angle $\phi$ (see Figure 1). In fact, it has been pointed out previously [23] that for a given radial displacement $\left(r_{0}\right)$ of an ion (point) source at the entrance $(x y)$ plane of the EIG, the maximal allowed initial elevation angle $\theta$ results from $\phi=\pi / 2$. Alternatively, for a given value of $r_{0}$ and $\theta$, the maximum allowed kinetic energy for which an ion may still be captured in a stable orbit about the wire electrode increases with increasing $\phi$ (up to $\phi=\pi / 2$, i.e., the ion velocity component perpendicular to the $z$ axis is also perpendicular to the direction of $r_{0}$ ). Figure 6 , 
Table 1. Collection efficiency (i.e., solid angle of the acceptance cone divided by $4 \pi$ ) for each of four combinations of ion initial kinetic energy $E_{\text {ion }}$ and potential difference $\Delta V$ between the cylinder $\left(r_{\text {cylinder }}=17 \mathrm{~mm}\right)$ and central wire $\left(r_{w i r e}=0.14 \mathrm{~mm}\right)$ of an electrostatic ion guide, for a circular disk ion source of 6 - $\mathrm{mm}$ radius and for a point ion source displaced radially by $6 \mathrm{~mm}$; both sources are located in the EIG entrance plarie

\begin{tabular}{|c|c|c|c|}
\hline$\Delta V(V)$ & $E_{\text {ion }}(\mathrm{eV})$ & $\begin{array}{c}\text { Eff }_{\text {disk }} \\
\left(r_{\text {d } 1 s k}=6 \mathrm{~mm}\right)\end{array}$ & $\begin{array}{c}\text { Eff } \\
\left(r_{\mathrm{o}}=6 \mathrm{~mm}\right)\end{array}$ \\
\hline 5.0 & 1.0 & 0.413 & 0.290 \\
\hline 10.0 & 2.5 & 0.331 & 0.232 \\
\hline 10.0 & 5.0 & 0.165 & 0.116 \\
\hline 5.0 & 5.0 & 0.083 & 0.058 \\
\hline
\end{tabular}

which is plotted for an ion that starts with $r_{0}=6 \mathrm{~mm}$, $\theta=15^{\circ}$, and $z_{0}=6 \mathrm{~mm}$, shows that ion initial kinetic energy is maximized for $130^{\circ} \leq \phi \leq 230^{\circ}$. In addition, note that as the ion source is moved away from the guide entrance plane, the kinetic energy maximum is achieved at higher azimuthal angle. In fact, with the ion source displaced by $\sim 15 \mathrm{~mm}$ (and other conditions as in Figure 6), the maximum kinetic energy (not shown) shifts to $\phi \approx 180^{\circ}$ (i.e., the ion velocity component perpendicular to $z$ points radially inward).

Comparison of the theoretical and observed maximum ion kinetic energies illustrates the importance of locating the ion source close to the EIG entrance plane. For example, under the same conditions as Figure 6 $\left(r_{0}=6 \mathrm{~mm}, \theta=15^{\circ}, \phi=0^{\circ}, \Delta V=10 \mathrm{~V}, E_{\mathrm{i} o n}=20\right.$ $\mathrm{eV})$, eq 7 yields a maximum ion kinetic energy of 31.6 $\mathrm{eV}$. As seen in Figure 6, the maximum kinetic energy of an ion that starts $6 \mathrm{~mm}$ axially displaced from the

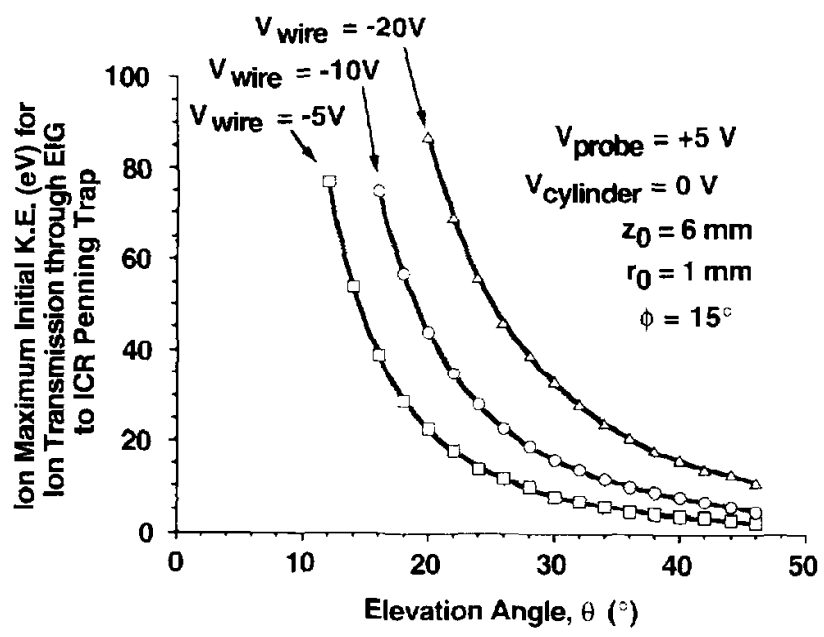

Figure 5. Maximum ion kinetic energy for EIG capture and ion transfer to an ICR Penning trap as a function of initial ion velocity elevation angle $\theta$ for an ion of $m / z 1000$ with initial axial and radial displacements of 1 and $6 \mathrm{~mm}$. The initial ion azimuthal angle $\phi$ is fixed at $15^{\circ}$. Higher ion kinetic energy or lower EIG potential difference results in decreased maximum acceptance angle, in qualitative agreement with eq 7 (which applies to the $z_{0}=0$ limit). entrance plane is $\sim 21 \mathrm{eV}$, or $\sim 33 \%$ less than that of an ion that starts at the EIG entrance plane $\left(z_{0}=0\right)$. Fortunately, the EIG cylinder diameter can be relatively large ( $34 \mathrm{~mm}$ in our case), so that any of several types of ion sources can be located conveniently at its entrance in an optimal geometric configuration.

\section{Ion Loss through Collision with the Central Wire}

A second advantage of injecting ions with large $\phi$ is the decrease in the number of ions that collide with the central wire electrode. Oakey and MacFarlane [23] have derived an expression for the minimum azimuthal angle $\phi_{\min }$ that an ion injected from a point source with a given $r_{0}$ and $\theta$ will be lost by collision with the wire:

$\phi_{\min }=\left(\frac{r_{\text {wire }}}{r_{0}}\right)\left(1+\left(\frac{q|\Delta V|}{E_{\text {ion }} \theta^{2}}\right)\left(\frac{\ln \left(r_{\text {wire }} / r_{0}\right)}{\ln \left(r_{\text {wire }} / r_{\text {cylinder }}\right)}\right)\right)^{1 / 2}$

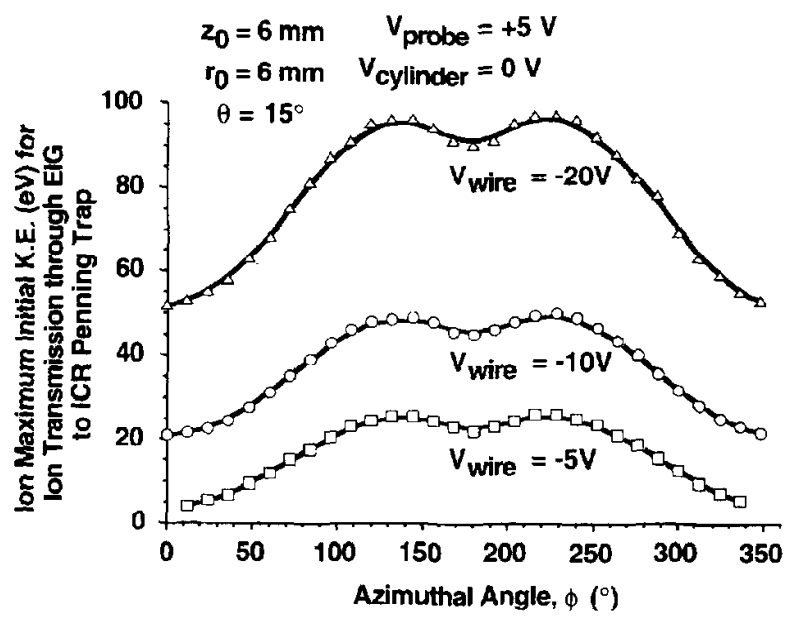

Figure 6. Maximum ion kinetic energy for EIG capture and ion transfer to an ICR Penning trap versus initial velocity azimuthal angle $\phi$ for an ion of $m / z 1000$ that starts $6 \mathrm{~mm}$ off-axis and 6 $\mathrm{mm}$ axially displaced from the EIG entrance plane. The ion initial velocity elevation angle $\theta$ is fixed at $15^{\circ}$. 
The fraction of ions that hit the wire as a function of ion initial radial displacement, $F_{\text {collide }}\left(r_{0}\right)$, is [23]

$$
\begin{aligned}
& F_{\text {collide }}\left(r_{0}\right) \\
& =\left(\frac{r_{\text {wire }}}{2 \pi r_{0}} \frac{q|\Delta V|}{E_{\mathrm{i} m}}\right) \\
& \times\left\{\left(\frac{\ln \left(r_{\text {(ytinder }} / r_{10}\right)}{\ln \left(r_{\text {(ylindere }} / r_{\text {wire }}\right)}\right)^{1 / 2}\right.
\end{aligned}
$$

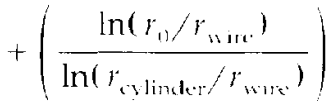

$$
\begin{aligned}
& \left.\times \ln \left\{\frac{+\left(\ln \left(r_{\text {(ylinder }} / r_{\text {wir }}\right)\right)^{1 / 2}}{\left(\ln \left(r_{0} / r_{w / r e}\right)\right)^{1 / 2}}\right)\right\}
\end{aligned}
$$

Combining eqs 8 and 11 yields the ratio $F_{\text {collide/capture }}\left(r_{0}\right)$ of the fraction of ions that are captured inside the EIG but collide with the central wire to the total fraction of ions captured, as a function of ion initial radial displacement [23]:

$$
\begin{aligned}
& F_{\text {collide/capture }}\left(r_{0}\right) \\
& =\left(\frac{2 r_{\text {wire }}}{\pi r_{0}}\right) \\
& \times\left\{\left(\frac{\ln \left(r_{\text {cylinder }} / r_{w i t}\right)}{\ln \left(r_{\text {(ulinder }} / r_{0}\right)}\right)^{12}+\left(\frac{\ln \left(r_{0} / r_{w \text { ire }}\right)}{\ln \left(r_{\text {cylinder }} / r_{0}\right)}\right)\right.
\end{aligned}
$$

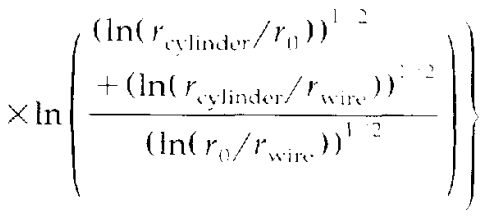

$$
\begin{aligned}
& x\left(1-\left(\frac{r_{0}^{2}}{r^{2} y_{\text {linder }}}\right)\right)^{\prime=}
\end{aligned}
$$

Based on the foregoing EIG dimensions, Table 2 lists values of $F_{\text {collide/apture }}\left(r_{0}\right)$, calculated from eq 12, for initial ion radial displacements of $1-10 \mathrm{~mm}$. Note that for a given set of ion initial conditions (e.g., $\Delta V, E_{\text {Iun }}$, $\phi$, and $\theta$ ), the fraction of ions that are lost to collision with the wire decreases with increasing initial radial displacement; however, even for relatively small initial ion radial displacement (e.g., $r_{0}=2 \mathrm{~mm}$ ), ion loss is only $\sim 10 \%$. Note that eqs $10-12$ apply for ions that begin at (or inside) the EIG entrance plane. Thus the values in Table 2 are estimates for ions that start out axially displaced from the entrance plane. Also, as discussed in succeeding text, the initial ion radial displacement also affects the ion final axial kinetic energy distribution at the ICR ion trap. The low number of ion-wire collisions coupled with a very large acceptance angle makes the EIG well suited to capture and transport a significant fraction of ions initially formed at the source

\section{Ion Transport through a Magnetic Field Gradient to an ICR Penning Trap}

As an ion passing through the EIG begins to enter the strong magnetic field region of the mass spectrometer, the ion velocity parallel to the magnetic field becomes [34]

$$
\mathbf{v}(z)=\mathbf{v}_{0}\left[1-\frac{\mathbf{B}(z)}{\mathbf{B}(0)} \sin ^{2} \theta\right]^{1 / 2}
$$

in which $\mathbf{v}_{0}$ is the ion initial velocity, $\mathbf{v}(z)$ is the ion axial velocity at position $z, \mathbf{B}(0)$ is the (axial) magnetic induction at the ion source, $\mathbf{B}(z)$ is the magnetic induction at position $z$, and $\theta$ is the ion initial elevation angle (see Figure 1) between $\mathbf{v}_{0}$ and $\mathbf{B}(0)$. For ions to pass through the magnetic mirror, an ion must maintain axial velocity greater than zero [i.e., $\mathbf{v}(z)>0$, so that the ion continues to move toward the ICR ion trap]. Kofel et al. [34] showed that in the absence of an electric field, ions will overcome the magnetic mirror effect provided that

$$
\theta<\arcsin \left[\frac{\mathbf{B}(0)}{\mathbf{B}(z)}\right]^{1 / 2}
$$

For values of $\theta$ above this critical limit, the mirror force dominates and ions are reflected away from the trap [i.e., $\mathbf{v}(z)<0$ ]. In the present experiments, $\mathbf{B}(0)$ at the source and $\mathbf{B}(z)$ at the ICR ion trap are approxi-

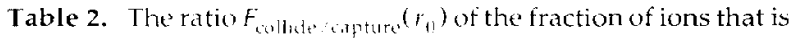
captured but collide with the EIG central wire to the total fraction of ions captured, as a function of initial ion radial displacement;

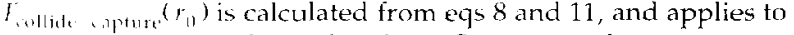
an ion point source located at the EIG entrance plane

\begin{tabular}{ll}
\hline$r_{0}(\mathrm{~mm})$ & $F_{\text {collidecapture }}\left(r_{0}\right)$ \\
\hline 1.0 & 0.18 \\
2.0 & 0.11 \\
3.0 & 0.084 \\
4.0 & 0.070 \\
5.0 & 0.060 \\
6.0 & 0.053 \\
7.0 & 0.048 \\
8.0 & 0.044 \\
9.0 & 0.040 \\
10.0 & 0.036 \\
\hline
\end{tabular}


mately 0.008 and $3 \mathrm{~T}$, respectively, which require that $\theta<3^{\circ}$ for transmission of ions that leave the source in the absence of the EIG. Thus a major advantage of the EIG lies in its large acceptance cone; even ions with large initial elevation angle at the ion source (see Figure 5) are held close to the $z$ axis and transported through the magnetic field gradient to the ICR cell.

\section{Ion Axial Kinetic Energy After Exiting the Flectrostatic Inn Guide}

Ions formed at the source with initial conditions ( $E_{\mathrm{ion}}, r_{0}, \theta, \phi$ ) have no rotational preference about the central wire because the magnetic field is negligible at the source. Conversely, once ions have passed through the magnetic field gradient to reach a homogeneous magnetic field, ions undergo cyclotron rotation in only one sense according to the right-hand rule for the Lorentz magnetic force, $\mathbf{F}=q \mathbf{v} \times \mathbf{B}$. Between the source and the homogeneous magnetic field region, ions that rotate inside the EIC in the same sense ("conrotatory") as the final ion cyclotron motion at the EIG exit will be axially decelerated (e.g., the mirror force $F_{z}=q r . B_{r}$ is directed along the negative $=$ axis) as they traverse the transition region between Lissajous-type motion (e.g., determined by the EIC potential difference) and cyclotron motion (e.g., determined strictly by the magnetic field). Alternatively, ions that initially rotate inside the EIG in the opposite sense ("disrotatory") as their final cyclotron motion will be axially acclerated in the - z direction in the region of high radial magnetic field. Because ion total kinetic energy must be conserved, any change in ion axial kinetic energy will be reflected in a corresponding change in the firlal ion cyclotron and magnetron radii.

Figure 7 shows the final ion cyclotron and mag netron radii $\rho_{+}$and $\rho$. (estimated from SIMION trajectory plots) as a function of ion initial azimuthal angle $\phi$ (with other initial conditions as in Figure 6). Ions that have $0^{\circ} \leq \phi \leq 180^{\circ}$ are disrotatory with respect to their final ICR motion. Moreover, note that the maximum $z$ acceleration and thus minimum cyclotron radius occurs at $\phi \approx 90^{\circ}$, which corresponds to the maximum initial angular momentum (see eq 7) with which an ion may be injected. Conversely, when an ion is injected with maximum conrotatory angular mo mentum $\left(\phi \approx 270^{\circ}\right)$, the final magnetron radius reaches a minimum (see Figure 7 ). Oyerall, the cyclotron and magnetron radii vary over the ranges $-1-6$ and 0.5-5 mm, respectively. The corresponding ion final axial kinetic energy, estimated by subtracting the ra dial kinetic energy (calculated from $\rho$, in Figure 7 ) from the total kinetic energy (provided by SIMION), varied over the range $-925 \mathrm{eV}$.

In Figures 6 and 7 , the final distribution in jon axial kinetic energy corresponds to ions for which $\theta=15$. In fact, ion sources will produce ions that have a range of $\theta$ values. Thus one might expect an even wider

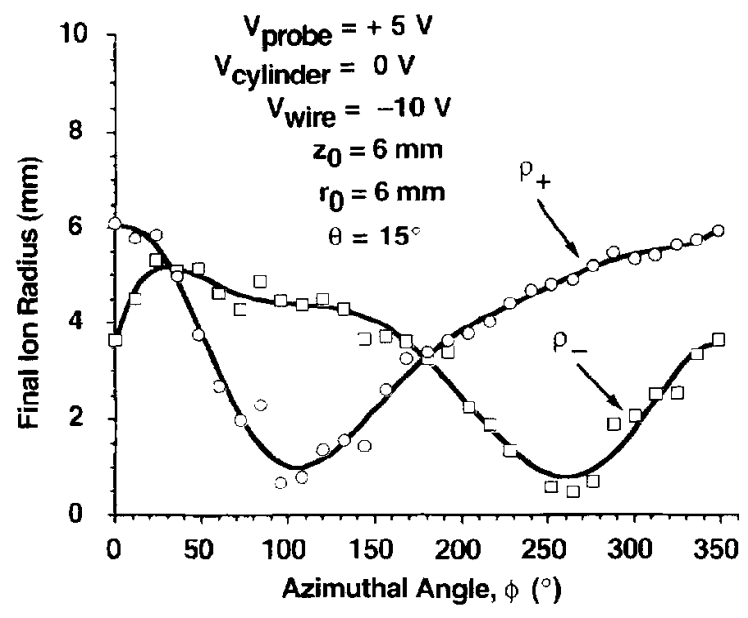

Figure 7. Final ion cyclotron and magnetron radii $\rho_{+}$and $\rho_{-}$ as a function of ion injtial velocity azimuthal angle $\phi$. lons begin with axial and radial displacements of $6 \mathrm{~mm}$ and velocity elevation angle $\theta$ fixed at $15^{\circ}$. Ions with $0^{\circ} \leq \phi \leq 180^{\circ}$ are "disrotatory," whereas those with $180^{\circ} \leq \phi \leq 360^{\circ}$ are "conrotatory" (see text 1 . The smooth curves simply represent polynomial fits to the calculated values.

range of final axial kinetic energies for ions formed with a wide range of initial $\theta$ and $\phi$ values. Figure 8 provides an estimate of the axial kinetic energy distribution for monoenergetic ions that have initial conditions as in Figure 6. For each elevation angle $\left(0 \rightarrow 20^{\circ}\right.$, in $2^{\circ}$ increments) the azimuthal angle was varied from $0 \rightarrow 360^{\circ}$ in $30^{\circ}$ increments. The shaded region represents an envelope that encompasses all of the data points. As expected, the acceleration-deceleration effects of the radial magnetic field result in a large axial kinetic energy distribution of approximately $20 \mathrm{eV}$. Thus, whereas the F.IC can transport a large fraction of ions that begin with their trajectories radially displaced from the $z$ axis, subsequent mass analysis is obviously complicated by the wide range of final ion axial kinetic energies. For example, the highest trapping efficiency is expected for narrow axial kinetic energy distributions, especially for accumulated trapping $[35,36]$, in which ions are collected for periods of up to several seconds prior to excitation and detection. Fortunately, the axial kinetic energy distribution of ions that exit the EIG may be narrowed simply by decreasing the ion initial radial displacement. Figure 9 displays results compiled as in Figure 8 for initial ion radial displacements of 2 (light shaded area) and $0 \mathrm{~mm}$ (dark shaded area). The axial kinetic energy distributions drop to -6 and $\sim 3 \mathrm{eV}$ for initial radial displacements of 2 and $0 \mathrm{~mm}$, respectively. Clearly, ions that begin their trajectories closer to the central wire will be held closer to the $z$ axis, thereby they encounter a smaller radial magnetic field and hence less axial acceleration or deceleration. Furthermore, ions that exit the EIG close to the central $z$ axis will have smaller cyclotron and magnetron radii, which facilitates subsequent mass analysis. Experimentally, we 


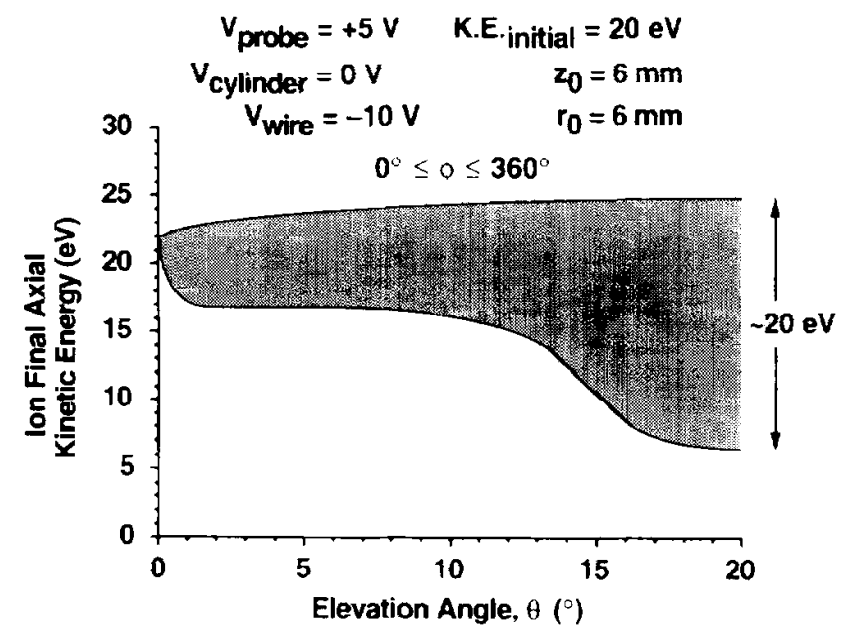

Figure 8. Final ion axial kinetic energy as a function of ion initial velocity elevation angle $t$. Ion initial conditions are as in Figures 6 and 7 , except that at cach $"$ valus, the initial velocity azimuthal angle is varied from $0 \mathrm{for} .3 \mathrm{fol}$ in 30 increments. Note. that the acceleration decoluation effects of the magnetic mirror result in a wide $\left(-2()_{-e}\right)$ ) axinl kinetic energs distribution (grop region).

have realized enhanced ion trapping efficiency and reduced postinjection ion cyclotron and magnetron radii by use of azimuthal quadrupolar excitation during ion injection on our prototype EIG system. Nevertheless, ion sources do not produce monoenergetic ions, and therefore the experimentally observed axial kinetic energy distribution will represent a convolution of the axial kinetic energy distribution inherent in the EIG (e.g., Figure 8 or 9) and the energy distribution of the ion source. Note that for ions with large initial radius $r_{0}$, increasing the EIC potential difference $\Delta V$ also will reduce the axjal kinctic distribution because ions then will execute Lissajous orbits closer to the wire electrode.

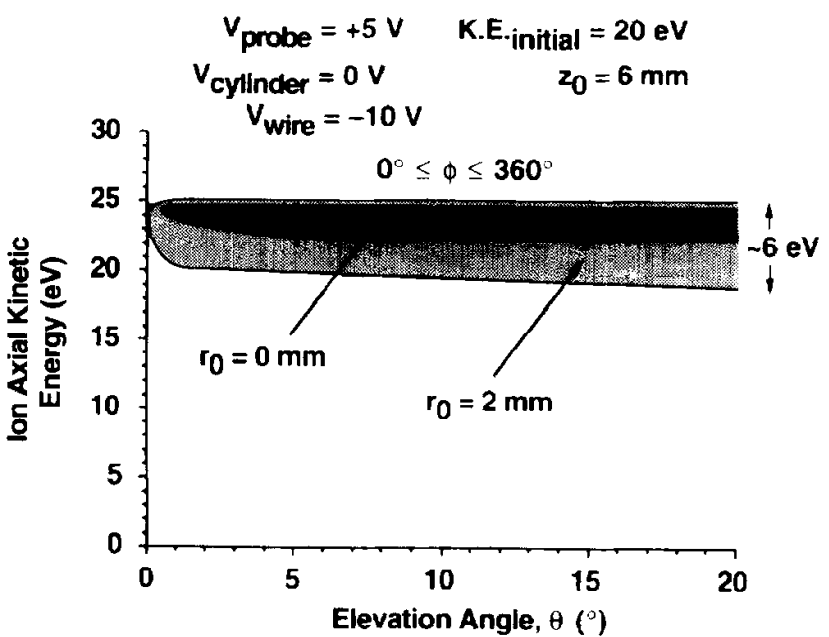

Figure 9. Final ion axial kinetic energy as a runction of jon initial velocity elevation angle $\|$, as in Figure 8 , for $r_{13}=2 \mathrm{~mm}$ (light shaded area) and $r_{11}=0 \mathrm{~mm}$ (dark shaded area). Note the narrowing of the final axial kinetic energr distribution as ions are focused closer to the $z$ axis.

\section{Limitations of the Present Trajectory Calculations}

Although the present results are for ions of a single mass-to-charge ratio $(m / z 1000)$ and a maximum magnetic field of $3 \mathrm{~T}$, we may nonetheless make several inferences for other experimental conditions. For example, in matrix-assisted laser desorption, ion kinetic energy is expected to scale linearly with mass because ion velocity is nearly mass independent (for ions of $m /=\geq 1000$ ) [37]. Based on an average ion velocity of $750 \mathrm{~m} / \mathrm{s}$ [37], our simulations (not shown) indicate that ions up to $m / z \approx 22,000\left(r_{0}=2 \mathrm{~mm}, z_{0}=6 \mathrm{~mm}\right.$, $\theta=15^{\circ}, \phi=45^{\circ}, L_{\mathrm{i} o \mathrm{n}} \approx 3-65 \mathrm{eV}$ ) are transported to the cell for a wire-cylinder potential difference of 10 $\checkmark$. Under these conditions, the maximum Lissajous orbital radius increased quadratically from $2.5(\mathrm{~m} / \mathrm{z}=$ $1000)$ to $15 \mathrm{~mm}(\mathrm{~m} / \mathrm{z}=22,000)$. As discussed in the preceding text, ions that have larger Lissajous radii will experience higher radial magnetic field and thus greater mirror effect. However, when the EIG potential difference was increased to $45 \mathrm{~V}$, the transmitted massto-charge ratio range extended above 100,000 (other conditions as before, with $E_{\text {icn }}$ extending to $\approx 300$ $\mathrm{eV}$ ), whereas the maximum Lissajous radius was reduced to $\leq 5 \mathrm{~mm}$ for $m / z \leq 40,000$ (again the Lissajous ractius increased quadratically to $15 \mathrm{~mm}$ for $40,000 \leq m / z \leq 100,000)$. Use of an EIG at higher magnetic field also will require larger wire and cylinder voltages because the radial magnetic field will be greater than in the present simulations. Note that other options exist to minimize the Lissajous orbital radius (and thus the deleterious effects of the radial magnetic field): For example, a smaller diameter wire would allow for smaller ion orbital radius during transport through the EIG. The use of a cylinder electrode that tapers from a relatively large diameter at the source region to a smaller diameter prior to the magnetic fringe field is a second intriguing approach. In this cise collection efficiency should be maximized at the EIC entrance, whereas ions would be squeezed close to the central axis prior to entering the region of high radial magnetic field.

The present analysis is predicated on assumed point or circular disk ion sources, each with uniform angular distribution. In fact, an actual ion source will have a nonuniform angular distribution; for example, a $\cos ^{2} \theta$ distribution for SIMS sources [38-41]. Moreover, the tiscrete nature of SIMION for both electric potential and magnetic field values necessarily limits the precision of the data. Finally, SIMION is limited to single-ion trajectories, so that ion-ion Coulomb forces and ion-neutral collisions are neglected. The presently reported EIG collection efficiencies thus probably represent upper limits in practice.

\section{Conclusions}

The present SIMION trajectory calculations predict that an electrostatic ion guide can transport, from an exter- 
nal ion source and through a magnetic field gradient into an ICR Penning trap, ions that have a wide range of initial kinetic energy and angular divergence. Three general aspects of EIG performance have been evaluated:

\section{Ion Capture}

The maximum kinetic energy for ion capture by the EIG is achieved by placing the ion source close to the EIG entrance plane and close to the EIG central axis. In addition, ion collection efficiency increases with increasing ratio of EIG potential difference to ion initial kinetic energy, $\Delta V / E_{\mathrm{i}, \mathrm{T}}$.

\section{Ion Transport}

Successful ion transport requires minimal ion interaction with the radial magnetic field. In this respect, small initial ion radial displacement and/or large $\Delta V$ will focus ions closer to the central axis, thereby reducing the magnetic mirror effect. However, ion collisions with the central wire electrode increase with decreasing $r_{0}$. Fortunately, Table 2 shows that only about $10 \%$ of ions collide with the central wire when the initial ion radial displacement is as small as $\sim 2 \mathrm{~mm}$.

\section{Ion Axial Kinetic Energy Distribution}

Independent of the efficiency of ion transport to the ICR Penning trap, efficient ion trapping requires that ions arrive with a narrow range of axial kinetic energies. The axial acceleration-deceleration effects of the magnetic field gradient may be minimized by small initial ion radial displacement (i.e., placing the ion source near the magnet z axis). Specifically, our trajectory simulations predict that an EIG with a cylinder-wire potential difference as low as $10 \mathrm{~V}$ should transport a large fraction of ions that have initial kinetic energies in the $4(1-50) \mathrm{eV}$ range, based on readily achievable ion source placement of $r_{0} \approx 2 \mathrm{~mm}$ and $z_{0} \approx 6 \mathrm{~mm}$, and ion initial angular divergence $\theta \leq 20^{\circ}$. Thus, the electrostatic ion guide appears to represent an attractive alternative to multipole guides and electrostatic lens systems.

\section{Acknowledgments}

We gratefully acknowledge S. Cuan and M. Wang for many helpful discussions. SIMION version 5.0 was generously provided by D. Dahl (Idaho National Fngineering Laboratory, ldaho Falls, ID). This work was supported by the National Science Foundation (CHE-9021058 and CHF-93-22824), the National Institutes of I lealth (GM-31683), The Ohio State University, and the National High Magnetic Field Laboratory at Florida State University. P.A.L.'s research was supported in part by the Oak Ridge Associated Universities under contract DE-AC05-760R00033 with the U.S. Department of Energs and by Louisiand State University.

\section{References}

1. Marshall, A. G.; Schweikhard, L. Int. J. Mass Spectrom. Ion Processes 1992, 118/119, 37-70.

2. Dunbar, R. C. Mass Spectrom. Rev. 1992, 11, 309-339.

3. Köster, C.; Kahr, M. S.; Castoro, J. A.; Wilkins, C. L. Mass Spectrom. Rev. 1992, 11, 495-512.

4. Speir, J. P.; Gorman, G. S.; Amster, I. J. In Mass Spectrometry in the Biological Scientes: A Tutorial; M. L. Gross, Ed.; Kluwer Academic Publishers: Dordrecht, The Netherlands, 1992; pp 199-212.

5. Jacoby, C. B.; Holliman, C. L.; Gross, M. L. In Mass Spectrometry in the Biological Sciences: A Tutorial; M. L. Gross, Ed.; Kluwer Academic Publishers: Dordrecht, 1992; pp 93-116.

6. Nibbering, N. M. M. Analyst 1992, 117, 289-293.

7. Schweikhard, L.; Marshall, A. G. I. Am. Soc. Mass Spectrom. 1993, 4, 433-452.

8. Buchanan, M. V; Hettich, R. L. Anal. Chem. 1993, 65, $245 \mathrm{~A}-259 \mathrm{~A}$

9. Brenna, J. T.; Creasy, W. R.; Zimmerman, I. Amer. Chem. Soc. Symp. Ser. 1993, 236, 129-154.

10. Guan, S.; Kim, H. S.; Marshall, A. G.; Wahl, M. C.; Wood, T. D.; Xiang, X. Chem. Rev. 1994, 8, 2161-2182.

11. Wilkins, C. L., Ed. Trends Ana. Chem. 1994, 13, 223-251. Fourier transform mass spectrometry; Special issue.

12. Holliman, C. L.; Rempel, D. L.; Gross, M. L. Mass Spectrom. Rev. 1994, 13, 105-132.

13. Guan, S.; Marshall, A. G. Int. I. Mass Spectrom. Ion Processes 1995, in press.

14. Limbach, P. A.; Marshall, A. G.; Wang, M. Int. J. Mass Spectrom. Ion Processes 1993, 125, 135-143.

15. Guan, S.; Paša-Tolić, L.; Marshall, A. G.; Xiang, X. Int. J. Mass Spectrom. Lon Processes 1994, 139, 75-86.

16. Jjames, C. F.; Markey, S. P. I. Am. Soc. Mass Spectrom. 1994, $5,398-406$.

17. Marto, J. A.; Guan, S.; Marshall, A. G. Rapid Commun. Mass Spectrom. 1994, 8, 615-620.

18. Huang, Y.; Guan, S.; Marshall, A. G. In Proceedings of the 42nd Antrican Suciety for Mass Spectronetry Conference on Mass Spectrometry and Alited Topics; Chicago, IL, 1994; $\mathrm{p} 239$.

19. Marto. J. A.; Guan, S.; Limbach, P. A.; Wahl, M. C.; Marshall, A. G. In Procedings of the 47st American Society for Mass Spectrometry Annual Conference on Mass Spectrometry and Allied Iopris; San Francisco, CA, 1993; pp 471a, b.

20. Limbach, P. A.; Marshall, A. G.; Duckworth, D. C.; Buchanan, M. V. In Procuedings of the 47st American Society for Mass Spectronetry Amnal Conference on Mass Spectrometry and Allied Toples; San Francisco, CA, 1993; pp 822a, b.

21. Mourad, W. G.; Pauly, T.; Heb, R. G. Rev. Sci. Instrum. 1964, $35,661-665$.

22. Herb, R. G.; Pauly, T,; Fisher, K. I. Bull. Am. Phys. Soc. 1963, 8,336 .

23. Oakey, N. S.; Macfarlane, R. D. Nucl. Instrum. Methods 1967, $49,220-228$

24. Abbe, J. C.; Amiel, S.; Macfarlane, R. D. Nucl. Instrum. Methods 1972, 702,73-76.

25. Igersheim, R.; Abbe, J. C.; Paulus, J. M.; Amiel, S. Nucl. Instrim. Mithods 1973, 109, 301-304.

26. Mactarlane, R. D.; Torgerson, D. F. Int. J. Mass Spectrom. Ion Phys. 1976, 21, 81-92.

27. Kenigsberg, A.; Leon, J.; Shafrir, N. H. Nucl. Instrum. Methods 1978, 148, 605612

28. Geno, P. W.; Macfarlane, R. D. Int. I. Mass Spectrom. Ion Proctsises 1986, 74, 43-57.

29. Wolf, B.; MacFarlane, R. D. J. Am. Soc. Mass Spectrom. 1992, $3,706-715$.

30. Hanson, C. D.; Just, C. L. And. Chem. 1994, 66, 3676-3680. 
31. Chen, F. F. Infroduction to Plasma Physics and Controlled Fusion, Vol. 1, 2nd ed.; Plenum Press: New York, 1984; Pf $30-34$.

32. Kingdon, K. H. Phys. Rev. 1923, 21, 408-418.

33. Johnson, C. E. /. Appl. Phys. 1984, 55, 3207-3214.

34. Kofel, P.; Allemann, M.; Kellerhals, H.; Wanczek, K.P. Imt. I. Mass Spectrom. Ion Processes 1986, 72, 53-61.

35. Beu, S. C.; Laude, D. A., Ir. Int. I. Mass Spectrom. Ion Processes 1991, 104, 109-127.

36. Beu, S. C.; Senko, M. W.; Quinn, I. P.; Wampler, F. M., III McLafferty, F. W. I. Amer. Soc Mass Spectrom. 1993, 4 $557-565$.

37. Beavis, R. C.; Chait, B. T. Chem. Ph1s. Lett. 1991, 181, 479 - 484.
38. Kelner, L.; Markey, S. P. Int. I. Mass Spectrom. Ion Processes 1984, 59, 157-167.

39. Kelner, L.; Patel, T. C. In Secondary Ion Mass Spectrometry: SIMS V, Vol. 44; A. Benninghoven, R. J. Colton, D. S. Simons, and H. W. Werner, Eds.; Springer-Verlag: Berlin, 1986; pp 494-496.

40. Benninghoven, A.; Rudenauer, F. G.; Werner, H. W. Secondary lon Mass Spectrometry, Vol. 86; Wiley: New York, 1987; p 1227.

41. Tsukakoshi, O.; Komatsu, K.; Komiya, S. In Secondary Ion Mass Spectrometry: SIMS IV, Vol. 36; A. Benninghoven, J. Okano, R. Shimizu, and H. W. Werner, Eds.; Springer-Verlag: Berlin, 1984; pp 70-72. 